\title{
TWISTOR SPACES AND THE ADIABATIC LIMITS OF DIRAC OPERATORS
}

\author{
MASAYOSHI NAGASE
}

\begin{abstract}
We show that a ( $\operatorname{Spin}^{q}$-style) twistor space admits a canonical Spin structure. The adiabatic limits of $\eta$-invariants of the associated Dirac operator and of an intrinsically twisted Dirac operator are then investigated.
\end{abstract}

\section{Introduction}

Let $\left(M, g^{M}\right)$ be an $n$-dimensional oriented Riemannian manifold equipped with a $\operatorname{Spin}^{q}$ structure introduced in [12]: $\operatorname{Spin}^{q}(n)=\operatorname{Spin}(n) \times_{\mathbb{Z}_{2}}$ $S p(1)$. Namely, the reduced structure bundle $P_{S O(n)}$ is assumed to have principal $\operatorname{Spin}^{q}(n)-, S O(3)$-bundles $P_{\operatorname{Spin}^{q}(n)}, P_{S O(3)}$ together with a $\operatorname{Spin}^{q}(n)$ equivariant bundle map

$$
\xi^{q}=\left(\xi_{0}^{q}, \xi_{1}^{q}\right): P_{\operatorname{Spin}^{q}(n)} \rightarrow P_{S O(n)} \times P_{S O(3)} .
$$

The concept came out from the idea of twisting Spin structure with $S p(1)$ to fit it with almost quaternionic structure and it exists if and only if the second Stiefel-Whitney class of $P_{S O(n)}$ is equal to the class of some $P_{S O(3)}$ : compare with the concept of $\mathrm{Spin}^{c}$ structure. Using the canonical action of $\operatorname{Spin}^{q}(n)$ on the quotient $\operatorname{Spin}^{q}(n) / \operatorname{Spin}^{c}(n)=S p(1) / U(1)=\mathbb{C} P^{1}$, then we get a $\mathbb{C} P^{1}$-fibration

$$
\pi: Z=P_{\operatorname{Spin}^{q}(n)} \times \text { can } \frac{\operatorname{Spin}^{q}(n)}{\operatorname{Spin}^{c}(n)} \rightarrow M,
$$

whose total space $Z$ is called a (Spin ${ }^{q}$-style) twistor space ([14]). Let us fix a connection $\alpha_{S O(3)}$ on $P_{S O(3)}$ and take the Levi-Civita connection $\alpha^{M}$ on $P_{S O(n)}$. By pulling back the product connection $\alpha^{M} \oplus \alpha_{S O(3)}$ by $\xi^{q}$, we obtain a connection $\alpha^{M, q}$ on $P_{\operatorname{Spin}^{q}(n)}$, which induces a splitting of the tangent bundle of $Z$ into horizontal and vertical components, $T Z=\mathcal{H} \oplus \mathcal{V}$.

Received October 29, 1999.

Revised August 17, 2000.

1991 Mathematics Subject Classification: Primary 57R15, 81R25, 53C27. 
The standard fibre $\mathbb{C} P^{1}$ has the Fubini-Study metric $d s^{2}$ (with holomorphic sectional curvature 1 ) and the canonical complex structure $J^{\mathbb{C} P^{1}}$, from which $\mathcal{V}$ inherits a hermitian complex line bundle structure $\left(d s^{\mathcal{V}}, J^{\mathcal{V}}\right)$. Now we denote by $g^{\mathcal{V}}$ the underlying Riemannian metric of $d s^{\mathcal{V}}$ and define a metric $g^{Z}$ on $Z$ by

$$
g^{Z}=\pi^{*} g^{M}+g^{\mathcal{V}}, \quad \pi^{*} g^{M}=g^{Z} \mid \mathcal{H}
$$

The reduced structure bundle $P_{S O(n+2)}(Z)$ of $\left(Z, g^{Z}\right)$ admits then a canonical $\operatorname{Spin}^{c}$ structure $([14, \S 1])$ :

$$
\xi^{c}=\left(\xi_{0}^{c}, \xi_{1}^{c}\right): P_{\operatorname{Spin}^{c}(n+2)}(Z) \rightarrow P_{S O(n+2)}(Z) \times P_{U(1)}(Z),
$$

which is constructed as follows: Since the map $P_{\operatorname{Spin}^{q}(n)} \rightarrow Z, p_{x} \mapsto\left[p_{x},[1]\right]$, obviously has a structure of principal $\operatorname{Spin}^{c}(n)$-bundle, we denote $P_{\operatorname{Spin}^{q}(n)}$ regarded as the total space of the bundle by $P_{\operatorname{Spin}^{c}(n)}(Z)$. This gives a $\operatorname{Spin}^{c}$ structure of $\pi^{*} P_{S O(n)}$, which is isomorphic by $\pi$ to the reduced structure bundle of $\left(\mathcal{H}, g^{Z} \mid \mathcal{H}\right)$

$$
\xi^{c}: P_{\operatorname{Spin}^{c}(n)}(Z) \rightarrow \pi^{*} P_{S O(n)} \times P_{U(1)}^{\mathcal{H}}(Z) .
$$

On the other hand, the reduced structure bundle $P_{S O(2)}^{\mathcal{V}}(Z)$ of $\left(\mathcal{V}, g^{\mathcal{V}}\right)$ has a canonical $\operatorname{Spin}^{c}$ structure ([11, Example D.6])

$$
\xi^{c}: P_{\operatorname{Spin}^{c}(2)}^{\mathcal{V}}(Z) \rightarrow P_{S O(2)}^{\mathcal{V}} \times P_{U(1)}^{\mathcal{V}}(Z)
$$

Here $P_{U(1)}^{\mathcal{V}}(Z)$ is the set of unitary frames of $\left(\mathcal{V}, d s^{\mathcal{V}}, J^{\mathcal{V}}\right)$. We may regard $\operatorname{Spin}^{c}(n)$ and $\operatorname{Spin}^{c}(2)$ as subgroups of $\operatorname{Spin}^{c}(n+2)$ through the inclusions $\mathbb{R}^{n}, \mathbb{R}^{2} \hookrightarrow \mathbb{R}^{n+2}$ and define a group homomorphism mult : $\operatorname{Spin}^{c}(n) \times$ $\operatorname{Spin}^{c}(2) \rightarrow \operatorname{Spin}^{c}(n+2)$ by multiplication in $\operatorname{Spin}^{c}(n+2)$. Then we set

(0.7) $P_{\operatorname{Spin}^{c}(n+2)}(Z)=\left(P_{\operatorname{Spin}^{c}(n)}(Z) \times P_{\operatorname{Spin}^{c}(2)}^{\mathcal{V}}(Z)\right) \times_{\text {mult }} \operatorname{Spin}^{c}(n+2)$.

Hence we have $P_{U(1)}(Z)=P_{U(1)}^{\mathcal{H}}(Z) \otimes P_{U(1)}^{\mathcal{V}}(Z)$.

Now the first purpose of the paper is to show

TheOREM 1. $\left(Z, g^{Z}\right)$ has a canonical Spin structure.

Here "canonical" means that it is a Spin structure

$$
\xi: P_{\operatorname{Spin}(n+2)}(Z) \rightarrow P_{S O(n+2)}(Z)
$$


which is uniquely determined (if it exists) by the condition that there is an isomorphism

$$
P_{\operatorname{Spin}^{c}(n+2)}(Z) \cong P_{\operatorname{Spin}(n+2)}(Z) \times_{\text {can }} \operatorname{Spin}^{c}(n+2) .
$$

Note that, in general, (0.5) and (0.6) cannot be reduced to Spin structures, that is, nontrivial double covering principal bundles $P_{\operatorname{Spin}(n)}(Z), P_{\operatorname{Spin}(2)}^{\mathcal{V}}(Z)$ of $\pi^{*} P_{S O(n)}, P_{S O(2)}^{\mathcal{V}}(Z)$ exist only locally. We will show, however, that we can take such locally defined bundles so successfully that the bundle $\left(P_{\operatorname{Spin}(n)}(Z) \times P_{\operatorname{Spin}(2)}^{\mathcal{V}}(Z)\right) \times{ }_{\text {mult }} \operatorname{Spin}(n+2)$ exists globally and gives exactly a canonical Spin structure.

Next, let us take spinor bundles $\mathbf{S}, \mathbf{S}^{c}$ associated to (0.8), (0.4). The connection $\alpha^{Z}$ induces a covariant derivative $\nabla^{\mathbf{S}}$ on $\mathbf{S}$, which defines a Dirac operator $D$ on it. Further the product connection made of $\alpha^{Z}$ and a connection $\alpha_{U(1)}$ on $P_{U(1)}(Z)([14, \S 2])$ induces a covariant derivative $\nabla^{\mathbf{S}^{c}}$ on $\mathbf{S}^{c}$, which defines a Dirac operator $D^{c}$ on it. Here $\alpha_{U(1)}$, which may not be trivial, on $P_{U(1)}(Z)$ which is trivial is a tensor product connection made of canonical ones $\alpha_{U(1)}^{\mathcal{H}}$ on $P_{U(1)}^{\mathcal{H}}(Z)$ and $\alpha_{U(1)}^{\mathcal{V}}$ on $P_{U(1)}^{\mathcal{V}}(Z)$, which are defined as follows: First, $P_{U(1)}^{\mathcal{H}}(Z)$ can be regarded as a subbundle of $\pi^{*} P_{S O(3)}$ because of a reduction embedding $P_{\operatorname{Spin}^{c}(n)}(Z) \equiv P_{\operatorname{Spin}^{q}(n)} \hookrightarrow \pi^{*} P_{\operatorname{Spin}^{q}(n)}$, $p_{x} \mapsto\left(\left[p_{x},[1]\right], p_{x}\right)$, and, moreover, $U(1)=S O(2)$ is naturally reductive in $S O(3)$, i.e., there is a natural splitting $\mathfrak{s o}(3)=\mathfrak{u}(1) \oplus \mathfrak{m}$ with $\operatorname{Ad}(U(1)) \mathfrak{m} \subset$ $\mathfrak{m}$. Hence the $\mathfrak{u}(1)$-component of $\pi^{*} \alpha_{S O(3)}$ restricted to $P_{U(1)}^{\mathcal{H}}(Z)$ gives its connection $\alpha_{U(1)}^{\mathcal{H}}$ : see $\S 3$. Second, let us denote the covariant derivative on $T Z$ associated to $\alpha^{Z}$ by $\nabla^{Z}$, which composed by the orthogonal projection $P^{\mathcal{V}}: T Z \rightarrow \mathcal{V}$ gives a covariant derivative $\nabla^{\mathcal{V}}=P^{\mathcal{V}} \nabla^{Z}$ on $\mathcal{V}$. The associated Ehresmann connection $\alpha^{\mathcal{V}}$ on $P_{S O(2)}^{\mathcal{V}}(Z)$ is then unitary with respect to $\left(d s^{\mathcal{V}}, J^{\mathcal{V}}\right)([14$, Lemma $2.1(4)])$ so that it induces a connection $\alpha_{U(1)}^{\mathcal{V}}$ on $P_{U(1)}^{\mathcal{V}}(Z)$.

Now, let us replace the metric $g^{Z}$ by

$$
g_{\varepsilon}^{Z}=\varepsilon^{-1} \pi^{*} g^{M}+g^{\mathcal{V}}, \quad \varepsilon>0
$$

and define Dirac operators $D_{\varepsilon}, D_{\varepsilon}^{c}$ accordingly. We restrict ourselves to the case $n$ is odd, that is, the case where their indices vanish, and want to investigate the limiting behavior of the $\eta$-invariants $\eta\left(D_{\varepsilon}\right), \eta\left(D_{\varepsilon}^{c}\right)$ when $\varepsilon \rightarrow 0$. The operation of blowing up the metric in the base space direction is called passing to the adiabatic limit. The idea of extracting some intrinsic 
values by taking the adiabatic limit is originally due to Witten [18], in which he found that, for a determinant line bundle associated to a family of certain invertible Dirac operators, the adiabatic limit of its $\eta$-invariant is related to the so-called global anomaly (or the holonomy). His result was given rigorous treatment in $[6,8]$ and further extended by BismutCheeger [5] and Dai [9], on which our investigation here depends. The second result of the paper is now stated as follows: Let us denote by $\Omega^{M}, \Omega^{\mathcal{V}}$ the curvature 2-forms of $\alpha^{M}, \alpha^{\mathcal{V}}$ and define the $\hat{A}$-genus forms $\hat{\mathbb{A}}\left(\Omega^{M}\right), \hat{\mathbb{A}}\left(\Omega^{\mathcal{V}}\right)$ by $\hat{\mathbb{A}}\left(\Omega^{M}\right)=\operatorname{det}^{1 / 2}\left(\left(\sqrt{-1} \Omega^{M} / 4 \pi\right) / \sinh \left(\sqrt{-1} \Omega^{M} / 4 \pi\right)\right)$ etc. Further, let us denote the curvature 2-forms of $\alpha_{U(1)}^{\mathcal{H}}, \alpha_{U(1)}^{\mathcal{V}}$ by $\Omega^{\mathcal{H}}, \Omega^{\mathcal{V}}$ which takes values in $\mathfrak{u}(1)$ in contrast to the above $\Omega^{\mathcal{V}}$, and define the first Chern forms $c_{1}\left(\Omega^{\mathcal{H}}\right)$, $c_{1}\left(\Omega^{\mathcal{V}}\right)$ by $c_{1}\left(\Omega^{\mathcal{H}}\right)=\operatorname{tr}\left(\sqrt{-1} \Omega^{\mathcal{H}} / 2 \pi\right)=\sqrt{-1} \Omega^{\mathcal{H}} / 2 \pi$ etc. Then we have

TheOREM 2. The (adiabatic) limits $\lim _{\varepsilon \rightarrow 0} \eta\left(D_{\varepsilon}\right)\left(\right.$ resp. $\left.\lim _{\varepsilon \rightarrow 0} \eta\left(D_{\varepsilon}^{c}\right)\right)$ exist and there are odd degree forms $\tilde{\eta}$ (resp. $\tilde{\eta}^{c}$ ) on $M$ such that

$$
\begin{gathered}
\lim _{\varepsilon \rightarrow 0} \eta\left(D_{\varepsilon}^{(c)}\right)=2 \int_{M} \hat{\mathbb{A}}\left(\Omega^{M}\right) \wedge \tilde{\eta}^{(c)}, \\
d \tilde{\eta}=\int_{Z / M} \hat{\mathbb{A}}\left(\Omega^{\mathcal{V}}\right), \\
d \tilde{\eta}^{c}=\int_{Z / M} \hat{\mathbb{A}}\left(\Omega^{\mathcal{V}}\right) \wedge \exp \left(\frac{1}{2} c_{1}\left(\Omega^{\mathcal{V}}\right)+\frac{1}{2} c_{1}\left(\Omega^{\mathcal{H}}\right)\right),
\end{gathered}
$$

where $\int_{Z / M}$ is the integral over the fibres.

This is certainly an extension of the comment offered in [14, Remark $5.2(2)]$, in which the limit of the reduced $\eta$-invariant $\bar{\eta}\left(D_{\varepsilon}^{c}\right)(\bmod \mathbb{Z})$ was investigated.

\section{$\S 1$. Some intrinsic bundles}

First let us briefly recall relevant facts on $\operatorname{Spin}, \operatorname{Spin}^{c}, \operatorname{Spin}^{q} \cdot \operatorname{Spin}(n)$ is a covering group of $S O(n)$ together with a short exact sequence

$$
1 \rightarrow \mathbb{Z}_{2} \rightarrow \operatorname{Spin}(n) \stackrel{\xi}{\rightarrow} S O(n) \rightarrow 1
$$

If $n \geq 3$ it is the universal one because $\pi_{1}(S O(n))=\mathbb{Z}_{2}$. Notice that the map (0.8) is equivariant to the homomorphism $\xi$ with $n$ replaced by $n+2$. 
Next, by twisting it with $U(1)$, we get $\operatorname{Spin}^{c}(n)=\operatorname{Spin}(n) \times_{\mathbb{Z}_{2}} U(1)$, which has a short exact sequence

$$
1 \rightarrow \mathbb{Z}_{2} \rightarrow \operatorname{Spin}^{c}(n) \stackrel{\xi^{c}}{\rightarrow} S O(n) \times U(1) \rightarrow 1,
$$

where $\xi^{c}([\varphi, z])=\left(\xi_{0}^{c}([\varphi, z]), \xi_{1}^{c}([\varphi, z])\right)=\left(\xi(\varphi), z^{2}\right)$. The maps $(0.4)-(0.6)$ are equivariant to the homomorphisms $\xi^{c}$ with $n$ replaced suitably. Further, by twisting it with the quaternionic unitary (or symplectic) group $S p(1)$, we obtain $\operatorname{Spin}^{q}(n)=\operatorname{Spin}(n) \times_{\mathbb{Z}_{2}} S p(1)$ together with a short exact sequence

$$
1 \rightarrow \mathbb{Z}_{2} \rightarrow \operatorname{Spin}^{q}(n) \stackrel{\xi^{q}}{\rightarrow} S O(n) \times S O(3) \rightarrow 1
$$

where $\xi^{q}([\varphi, \lambda])=\left(\xi_{0}^{q}([\varphi, \lambda]), \xi_{1}^{q}([\varphi, \lambda])\right)=(\xi(\varphi), \operatorname{Ad}(\lambda))$. Here $\mathbb{R}^{3}$ and the Lie algebra $\mathfrak{s p}(1)=\operatorname{Im} \mathbb{H}=\left\{a_{1} \mathbf{i}+a_{2} \mathbf{j}+a_{3} \mathbf{k} \mid a_{\ell} \in \mathbb{R}\right\}\left(\mathbf{i}^{2}=\mathbf{j}^{2}=\mathbf{k}^{2}=-1\right.$, $\mathbf{i j}=-\mathbf{j i}=\mathbf{k}$ ), are naturally identified and the homomorphism Ad is defined by $S O(3)=S O(\mathfrak{s p}(1)) \ni \operatorname{Ad}(\lambda): a \mapsto \lambda a \lambda^{-1}$.

Now, let us take the standard representation of $S p(1)$

$$
\begin{aligned}
& r_{H}: S p(1) \rightarrow\left(G L_{\mathbb{H}}(\mathbb{H}) \hookrightarrow\right) G L_{\mathbb{C}}\left(\mathbb{C}^{2}\right) \equiv G L_{\mathbb{C}}(H), \\
& r_{H}(\xi+\mathbf{j} \eta)=\left(\begin{array}{rr}
\xi & -\bar{\eta} \\
\eta & \bar{\xi}
\end{array}\right)
\end{aligned}
$$

and define a locally defined vector bundle

$$
\mathbf{H}=P_{\operatorname{Spin}^{q}(n)} \times_{r_{H}} H \rightarrow M .
$$

To explain it more explicitly, we will fix local trivializations $P_{\operatorname{Spin}^{q}(n)} \mid U_{a} \cong$ $U_{a} \times \operatorname{Spin}^{q}(n), p_{x} \leftrightarrow\left(x, \tilde{f}_{a}\left(p_{x}\right)\right)$, over an open covering $\left\{U_{a}\right\}$ of $M$ and set $\tilde{f}_{b a}(x)=\tilde{f}_{b}\left(p_{x}\right) \tilde{f}_{a}\left(p_{x}\right)^{-1}$, which form a family of transition functions. Take a family $\left\{\tilde{f}_{1 b a}: U_{a} \cap U_{b} \rightarrow S p(1)\right\}\left(\tilde{f}_{1 a a} \equiv 1\right)$ with $\tilde{f}_{b a}=\left[\tilde{f}_{0 b a}, \tilde{f}_{1 b a}\right]$. On $U_{a} \cap U_{b} \cap U_{c}(\neq \emptyset), \tilde{f}_{1 c b} \tilde{f}_{1 b a}$ may differ in sign from $\tilde{f}_{1 c a}$. Note that such ambiguity cannot be removed in general. (1.5) is now a "vector bundle" associated to the family of, to say, pseudo-transition functions $\left\{\tilde{f}_{1 b a}\right\}$. It will be obvious then that the associated projective bundle is globally defined and coincides with $(0.2)$,

$$
\pi: Z=P(\mathbf{H}) \equiv P_{\operatorname{Spin}^{q}(n)} \times_{r_{H}} P(H) \rightarrow M .
$$

Let us consider next a locally defined tautological (or universal) line bundle

$$
\mathbf{U}_{Z}=\left\{([f], c f) \in \pi^{*} \mathbf{H}\right\} \rightarrow P(\mathbf{H})=Z .
$$


We will show that the tensor bundle $\mathbf{U}_{Z} \otimes \mathbf{U}_{Z}$ is canonically isomorphic to the relative canonical line bundle (or the family of canonical line bundles along the fibres)

$$
K_{Z} \rightarrow Z
$$

Note that we have $K_{Z} \mid \pi^{-1}(x)=\wedge^{1,0}\left(\left(\mathcal{V}, J^{\mathcal{V}}\right)^{*} \mid \pi^{-1}(x)\right) \cong \wedge^{1,0}\left(T_{\mathbb{C}}^{*} \mathbb{C} P^{1}\right)$ naturally. Let us denote canonical local coordinates of $P(H)=\mathbb{C} P^{1}$ on $W_{\ell}=\left\{\left[z_{0}, z_{1}\right] \mid z_{\ell} \neq 0\right\}$ by $w_{\ell}\left(=z_{1} / z_{0}(\ell=0), z_{0} / z_{1}(\ell=1)\right)$ and take local cross-sections of $\mathbf{U}_{Z} \mid \pi^{-1}(x)$,

$$
u_{\ell}: W_{\ell} \rightarrow W_{\ell} \times H
$$

defined by $u_{\ell}\left(w_{\ell}\right)=\left(w_{0},\left(1, w_{0}\right)\right)(\ell=0),\left(w_{1},\left(w_{1}, 1\right)\right)(\ell=1)$. The following short sequence is exact on each fibre $\pi^{-1}(x)([16,(2.7)])$ :

$$
0 \rightarrow \mathbf{U}_{Z} \rightarrow \pi^{*} \mathbf{H} \rightarrow \mathbf{U}_{Z} \otimes K_{Z}^{*} \rightarrow 0
$$

where the map $\pi^{*} \mathbf{H} \rightarrow \mathbf{U}_{Z} \otimes K_{Z}^{*}$ is given by $\left(w_{0},(\alpha, \beta)\right) \mapsto\left(w_{0},\left(-\alpha w_{0}+\right.\right.$ $\left.\beta) u_{0} \otimes \partial / \partial w_{0}\right),\left(w_{1},(\alpha, \beta)\right) \mapsto\left(w_{1},\left(\alpha-\beta w_{1}\right) u_{1} \otimes \partial / \partial w_{1}\right)$. Accordingly we can identify $\pi^{*} \mathbf{H} \cong \mathbf{U}_{Z} \oplus \mathbf{U}_{Z}^{\perp} \cong \mathbf{U}_{Z} \oplus\left(\mathbf{U}_{Z} \otimes K_{Z}^{*}\right)$ on each fiber, which yields global identifications $\mathbf{U}_{Z} \otimes \mathbf{U}_{Z} \otimes K_{Z}^{*} \cong \wedge \pi^{*} \mathbf{H} \cong \pi^{*} \wedge \mathbf{H} \cong \mathbb{C}_{Z}$ (trivial). Hence we have

LEMMA 1.1. We have

$$
K_{Z} \cong \mathbf{U}_{Z} \otimes \mathbf{U}_{Z}
$$

whose isomorphism is given by $d w_{0} \leftrightarrow u_{0} \otimes u_{0}, d w_{1} \leftrightarrow-u_{1} \otimes u_{1}$.

\section{$\S 2$. Proof of Theorem 1}

First, let us show that the line bundle

$$
\mathbf{L}_{\mathcal{H}}=P_{U(1)}^{\mathcal{H}}(Z) \times_{\text {can }} \mathbb{C}=P_{\operatorname{Spin}^{c}(n)}(Z) \times_{\xi_{1}^{c}} \mathbb{C}
$$

is canonically isomorphic to $K_{Z}$.

We begin with recalling transition functions of $P_{\operatorname{Spin}^{c}(n)}(Z)([14,(1.19)])$. The bundle $\operatorname{Spin}^{q}(n) \rightarrow \operatorname{Spin}^{q}(n) / \operatorname{Spin}^{c}(n)=\mathbb{C} P^{1}$ has a local cross-section $f_{0}=\left[1, \rho_{0}\right]$ over $W_{0}$ with

$$
\rho_{0}\left(w_{0}\right)=\rho_{0}\left(\left[1+\mathbf{j} w_{0}\right]\right)=\left(1+\mathbf{j} w_{0}\right) /\left|1+\mathbf{j} w_{0}\right|
$$


and $f_{1}=\left[1, \rho_{1}\right]$ over $W_{1}=\mathbf{j} W_{0}$ with $\rho_{1}\left(w_{1}\right)=\rho_{1}\left(\left[w_{1}+\mathbf{j}\right]\right)=\mathbf{j} \rho_{0}\left(\mathbf{j}^{-1}\left[w_{1}+\right.\right.$ $\mathbf{j}])=\left(w_{1}+\mathbf{j}\right) /\left|w_{1}+\mathbf{j}\right|$. Consider the local trivializations $\pi^{-1}\left(U_{a}\right) \cong U_{a} \times \mathbb{C} P^{1}$ of the bundle (0.2) which are induced from those of $P_{\operatorname{Spin}^{q}(n)}$ given in $\S 1$. Denote by $U_{a \ell}$ the open sets of $Z$ corresponding to $U_{a} \times W_{\ell}\left(\subset U_{a} \times \mathbb{C} P^{1}\right)$. Then we obtain local trivializations $P_{\operatorname{Spin}^{c}(n)}(Z) \mid U_{a \ell} \cong U_{a} \times W_{\ell} \times \operatorname{Spin}^{c}(n)$, $p_{x} \leftrightarrow\left(x, w_{\ell}, f_{\ell}\left(w_{\ell}\right)^{-1} \tilde{f}_{a}\left(p_{x}\right)\right)\left(P_{\operatorname{Spin}^{c}(n)}(Z) \equiv P_{\operatorname{Spin}^{q}(n)} \ni p_{x}\right.$ over $x \in M$, $\left.w_{\ell}=\left[\tilde{f}_{a}\left(p_{x}\right)\right] \in W_{\ell} \subset \mathbb{C} P^{1}\right)$, and the associated transition functions over $U_{a \ell} \cap U_{b \ell^{\prime}}\left(\ni z_{x}=\left(x, w_{\ell}\right) \in U_{a} \times W_{\ell}\right)$ are

$$
\psi_{\left(b \ell^{\prime}\right)(a \ell)}\left(x, w_{\ell}\right)=f_{\ell^{\prime}}\left(\tilde{f}_{b a}(x) f_{\ell}\left(w_{\ell}\right)\right)^{-1} \tilde{f}_{b a}(x) f_{\ell}\left(w_{\ell}\right) .
$$

This implies that (2.1) has a family of transition functions

$$
\xi_{1}^{c}\left(\psi_{\left(b \ell^{\prime}\right)(a \ell)}\left(x, w_{\ell}\right)\right)=\left(\rho_{\ell^{\prime}}\left(\tilde{f}_{1 b a}(x) \rho_{\ell}\left(w_{\ell}\right)\right)^{-1} \tilde{f}_{1 b a}(x) \rho_{\ell}\left(w_{\ell}\right)\right)^{2}
$$

over $U_{a \ell} \cap U_{b \ell^{\prime}}$.

On the other hand, if we trivialize $\mathbf{U}_{Z} \mid U_{a \ell}$ using $u_{\ell} /\left|u_{\ell}\right|$, the associated pseudo-transition functions over $U_{a \ell} \cap U_{b \ell^{\prime}}$ are certainly given by

$$
\psi_{1\left(b \ell^{\prime}\right)(a \ell)}\left(x, w_{\ell}\right)=\rho_{\ell^{\prime}}\left(\tilde{f}_{1 b a}(x) \rho_{\ell}\left(w_{\ell}\right)\right)^{-1} \tilde{f}_{1 b a}(x) \rho_{\ell}\left(w_{\ell}\right) .
$$

Actually, we have $u_{\ell}\left(w_{\ell}\right) /\left|u_{\ell}\left(w_{\ell}\right)\right|=\rho_{\ell}\left(w_{\ell}\right) \in H=\mathbb{H}$ and the two cross-sections $\rho_{\ell}\left(z_{x}\right) t, \rho_{\ell^{\prime}}\left(z_{x}\right) t^{\prime}$ of $\mathbf{U}_{Z}$ over $U_{a \ell}, U_{b \ell^{\prime}}$ are equivalent to each other if $\tilde{f}_{1 b a}(x) \rho_{\ell}\left(z_{x}\right) t=\rho_{\ell^{\prime}}\left(z_{x}\right) t^{\prime}$ holds on $U_{a \ell} \cap U_{b \ell^{\prime}}$. Thus we have $t^{\prime}=$ $\rho_{\ell^{\prime}}\left(z_{x}\right)^{-1} \tilde{f}_{1 b a}(x) \rho_{\ell}\left(z_{x}\right) t=\psi_{1\left(b \ell^{\prime}\right)(a \ell)}\left(x, w_{\ell}\right) t$.

Now (2.4), (2.5) and Lemma 1.1 imply

\section{LEMMA 2.1. We have}

$$
\mathbf{L}_{\mathcal{H}} \cong \mathbf{U}_{Z} \otimes \mathbf{U}_{Z} \cong K_{Z}
$$

whose isomorphisms are given by

$$
\left[p_{x}, t\right]=\left[\left(x, w_{\ell}, 1\right), t\right] \leftrightarrow \frac{t}{1+\left|w_{\ell}\right|^{2}} u_{\ell} \otimes u_{\ell} \leftrightarrow \frac{ \pm t}{1+\left|w_{\ell}\right|^{2}} d w_{\ell}
$$

over $U_{a \ell}$.

On the other hand, the line bundle

$$
\mathbf{L}_{\mathcal{V}}=P_{U(1)}^{\mathcal{V}}(Z) \times_{\text {can }} \mathbb{C}
$$


is obviously equal to $K_{Z}^{*}$ so that

$$
\mathbf{L}=P_{U(1)}(Z) \times_{\operatorname{can}} \mathbb{C}=\mathbf{L}_{\mathcal{H}} \otimes \mathbf{L}_{\mathcal{V}}
$$

is certainly trivial. Thus we have $w_{2}\left(P_{S O(n+2)}(Z)\right) \equiv c_{1}(\mathbf{L})=0$, which implies that $\left(Z, g^{Z}\right)$ admits a Spin structure. In the following, let us construct a canonical one concretely.

Recall that we have fixed a family of pseudo-transition functions $\tilde{f}_{1 b a}$ of (1.5), which defines the locally defined bundles $\mathbf{U}_{Z}, \mathbf{U}_{Z}^{*}$ and further defines the reduced structure bundles $P_{U(1)}\left(\mathbf{U}_{Z}\right), P_{U(1)}\left(\mathbf{U}_{Z}^{*}\right)$ : see (2.5). Here we will consider a family of their counterparts $\tilde{f}_{0 b a}$, i.e., $\tilde{f}_{b a}=\left[\tilde{f}_{0 b a}, \tilde{f}_{1 b a}\right]$. It forms a family of pseudo-transition functions, which defines a locally defined $\operatorname{Spin}(n)$-bundle $P_{\operatorname{Spin}(n)}$ over $M$. By pulling it back, we obtain a locally defined $\operatorname{Spin}(n)$-bundle $P_{\operatorname{Spin}(n)}$ over $Z$

$$
P_{\mathrm{Spin}(n)}(Z)=\pi^{*} P_{\mathrm{Spin}(n)} .
$$

It will be clear now that we can identify

$$
P_{\operatorname{Spin}^{c}(n)}(Z)=P_{\operatorname{Spin}(n)}(Z) \times_{\mathbb{Z}_{2}} P_{U(1)}\left(\mathbf{U}_{Z}\right)
$$

Refer to (2.3) and (2.5).

Next, let us consider pseudo-transition functions $\bar{\psi}_{1\left(b \ell^{\prime}\right)(a \ell)}$, the conjugates of $\psi_{1\left(b \ell^{\prime}\right)(a \ell)}$ given in $(2.5)$. These give a family of pseudo-transition functions

$$
\begin{aligned}
\bar{\psi}_{1\left(b \ell^{\prime}\right)(a \ell)}^{\mathbb{R}}=\operatorname{Re} \bar{\psi}_{1\left(b \ell^{\prime}\right)(a \ell)}+\operatorname{Im} \bar{\psi}_{1\left(b \ell^{\prime}\right)(a \ell)} \cdot e_{1}^{\prime \prime} \circ e_{2}^{\prime \prime} \\
: U_{a \ell} \cap U_{b \ell^{\prime}} \rightarrow \operatorname{Spin}(2),
\end{aligned}
$$

where $\left\{e_{1}^{\prime \prime}, e_{2}^{\prime \prime}\right\}$ are the standard basis of $\mathbb{R}^{2}$. It defines now a locally defined $\operatorname{Spin}(2)$-bundle $P_{\operatorname{Spin}(2)}^{\mathcal{V}}(Z)$. Recall that we have

$$
P_{\operatorname{Spin}^{c}(2)}^{\mathcal{V}}(Z)=P_{U(1)}\left(K_{Z}^{*}\right) \times \Xi^{c} \operatorname{Spin}^{c}(2)
$$

Here $\Xi^{c}$ is a homomorphism $U(1) \rightarrow \operatorname{Spin}^{c}(2)$ given by $e^{\sqrt{-1} \theta} \mapsto[\cos (\theta / 2)$ $\left.+\sin (\theta / 2) e_{1}^{\prime \prime} \circ e_{2}^{\prime \prime}, e^{\sqrt{-1} \theta / 2}\right]([11$, Example D.6] or $[14,(1.13)-(1.14)])$ and $P_{U(1)}\left(K_{Z}^{*}\right)$ is the reduced structure bundle of $K_{Z}^{*}$. It will be thus apparent that we can identify as follows:

$$
P_{\operatorname{Spin}^{c}(2)}^{\mathcal{V}}(Z)=P_{\operatorname{Spin}(2)}^{\mathcal{V}}(Z) \times_{\mathbb{Z}_{2}} P_{U(1)}\left(\mathbf{U}_{Z}^{*}\right)
$$


Now we set

$$
P_{\operatorname{Spin}(n+2)}(Z)=\left(P_{\operatorname{Spin}(n)}(Z) \times P_{\operatorname{Spin}(2)}^{\mathcal{V}}(Z)\right) \times{ }_{\text {mult }} \operatorname{Spin}(n+2),
$$

which is globally defined. Actually, the pseudo-transition functions $\pi^{*} \tilde{f}_{0 b a}$ of $P_{\operatorname{Spin}(n)}(Z)$ and $\bar{\psi}_{1\left(b \ell^{\prime}\right)(a \ell)}^{\mathbb{R}}$ (made from $\tilde{f}_{1 b a}$ ) of $P_{\operatorname{Spin}(2)}^{\mathcal{V}}(Z)$ have ambiguity, which, however, is removed fortunately by taking their multiplication. The splitting $T Z=\mathcal{H} \oplus \mathcal{V}$ implies then

$$
\begin{aligned}
P_{S O(n+2)}(Z) & =\left(\pi^{*} P_{S O(n)} \times P_{S O(2)}^{\mathcal{V}}(Z)\right) \times_{\text {mult }} S O(n+2) \\
& =P_{\operatorname{Spin}(n+2)}(Z) \times_{\xi} S O(n+2) .
\end{aligned}
$$

Thus we obtain a Spin structure of $\left(Z, g^{Z}\right)$. Further (2.11) and (2.14) imply canonically

$$
\begin{aligned}
P_{\operatorname{Spin}^{c}(n+2)}(Z) & =P_{\operatorname{Spin}(n+2)}(Z) \times_{\mathbb{Z}_{2}} P_{U(1)}\left(\mathbf{U}_{Z}\right) \otimes P_{U(1)}\left(\mathbf{U}_{Z}^{*}\right) \\
& =P_{\operatorname{Spin}(n+2)}(Z) \times_{\mathbb{Z}_{2}} U(1)_{Z}
\end{aligned}
$$

where $U(1)_{Z}$ is the trivial $U(1)$-bundle over $Z$. Thus we can regard $P_{\operatorname{Spin}(n+2)}(Z)$ naturally as a subbundle of $P_{\operatorname{Spin}^{c}(n+2)}(Z)$ so that we get an identification (0.9).

Finally, since the set of Spin structures of $P_{S O(n+2)}(Z)$ naturally corresponds bijectively to the set of $\operatorname{Spin}^{c}$ structures of $P_{S O(n+2)}(Z) \times U(1)_{Z}$ (through $H^{1}\left(Z, \mathbb{Z}_{2}\right)$ ), a Spin structure (0.8) with (0.9) exists uniquely.

\section{$\S$ 3. The covariant derivative $\nabla^{\mathbf{L}_{\mathcal{H}}}$ on $\mathbf{L}_{\mathcal{H}}$ associated to $\alpha_{U(1)}^{\mathcal{H}}$}

Let us denote by $\alpha_{S O(3)}^{a}$ the connection forms of $\alpha_{S O(3)}$ associated to local trivializations $P_{S O(3)}\left|U_{a}=\left(P_{\operatorname{Spin}^{q}(n)} \times_{\xi_{1}^{q}} S O(3)\right)\right| U_{a} \cong U_{a} \times S O(3)$, $\left[p_{x}, 1\right] \leftrightarrow \varphi_{a}\left(\left[p_{x}, 1\right]\right)=\left(x, \xi_{1}^{q}\left(\tilde{f}_{a}\left(p_{x}\right)\right)\right)$. Namely, we take a cross-section $\sigma_{a}$ : $U_{a} \rightarrow P_{S O(3)} \mid U_{a}$ with $\sigma_{a}(x)=\varphi_{a}^{-1}(x, 1)$ and put $\alpha_{S O(3)}^{a}=\sigma_{a}^{*} \alpha_{S O(3)}$. Take the standard basis $\left\{v_{1}, v_{2}, v_{3}\right\}=\{\mathbf{i}, \mathbf{j}, \mathbf{k}\}$ of $\mathbb{R}^{3}=\mathfrak{s p}(1)$ and set $\left(v_{i} \wedge v_{j}\right)(v)=$ $\left\langle v_{i}, v\right\rangle v_{j}-\left\langle v_{j}, v\right\rangle v_{i}$, where $\langle$,$\rangle is the standard inner product of \mathbb{R}^{3}$. Then $\left\{v_{i} \wedge v_{j}\right\}_{i<j}$ is a basis of $\mathfrak{s o}(3)$. Accordingly we may put

$$
\begin{aligned}
\alpha_{S O(3)}^{a} & =\sum_{i<j}\left[\alpha_{S O(3)}^{a}\right]_{j i} v_{i} \wedge v_{j} \\
& =\alpha_{32}^{a} v_{2} \wedge v_{3}+\alpha_{13}^{a} v_{3} \wedge v_{1}+\alpha_{21}^{a} v_{1} \wedge v_{2} .
\end{aligned}
$$


Next, let us take local trivializations $\pi^{*} P_{\text {Spin }^{q}(n)} \mid U_{a \ell} \cong U_{a} \times W_{\ell} \times$ $\operatorname{Spin}^{q}(n),\left(z_{x}, q_{x}\right) \leftrightarrow\left(x, w_{\ell}, f_{\ell}\left(w_{\ell}\right)^{-1} \tilde{f}_{a}\left(q_{x}\right)\right)$, where $z_{x}=\left[p_{x},[1]\right]$ belongs to $Z \mid \pi^{-1}(x), q_{x}$ is a point on the fibre of $P_{\operatorname{Spin}^{q}(n)}$ over $x$ and we set $w_{\ell}=\left[\tilde{f}_{a}\left(p_{x}\right)\right] \in W_{\ell} \subset \mathbb{C} P^{1}$. Then $\pi^{*} P_{S O(3)}=\pi^{*} P_{\operatorname{Spin}^{q}(n)} \times_{\xi_{1}^{q}} S O(3)$ has local trivializations $\left(\pi^{*} P_{S O(3)}\right) \mid U_{a \ell} \cong U_{a} \times W_{\ell} \times S O(3),\left[\left(z_{x}, q_{x}\right), 1\right]$ $\leftrightarrow\left(x, w_{\ell}, \xi_{1}^{q}\left(f_{\ell}\left(w_{\ell}\right)^{-1} \tilde{f}_{a}\left(q_{x}\right)\right)\right)$. As for the associated connection forms $\left(\pi^{*} \alpha_{S O(3)}\right)^{a \ell}$ of the connection $\pi^{*} \alpha_{S O(3)}$, we have

LEMmA 3.1. Take $X \in T_{x} U_{a}$ and $V \in T_{w_{\ell}} W_{\ell}$, which we regard as elements of $T_{x} U_{a} \times T_{w_{\ell}} W_{\ell}=T_{\left(x, w_{\ell}\right)} U_{a \ell}$. Then we have

$$
\begin{aligned}
& \left(\pi^{*} \alpha_{S O(3)}\right)^{a \ell}(X)=\operatorname{Ad}\left(\xi_{1}^{q}\left(f_{\ell}\left(w_{\ell}\right)\right)^{-1}\right) \alpha_{S O(3)}^{a}(X), \\
& \left(\pi^{*} \alpha_{S O(3)}\right)^{a \ell}(V)=\xi_{1}^{q}\left(f_{\ell}\left(w_{\ell}\right)\right)^{-1} d\left(\xi_{1}^{q} \circ f_{\ell}\right)(V) .
\end{aligned}
$$

Proof. Set $X=(d / d t)_{t=0}\left(x_{t}, w_{\ell}\right)$ with $x_{0}=x$. Then we have

$$
\begin{aligned}
& \left(\pi^{*} \alpha_{S O(3)}\right)^{a \ell}(X)=\alpha_{S O(3)}\left((d / d t)_{t=0} \varphi_{a}^{-1}\left(x_{t}, \xi_{1}^{q}\left(f_{\ell}\left(w_{\ell}\right)\right)\right)\right) \\
& =\alpha_{S O(3)}\left(R_{\xi_{1}^{q}\left(f_{\ell}\left(w_{\ell}\right)\right) *} \sigma_{a *} X\right)=\operatorname{Ad}\left(\xi_{1}^{q}\left(f_{\ell}\left(w_{\ell}\right)\right)^{-1}\right) \alpha_{S O(3)}^{a}(X) .
\end{aligned}
$$

Further, set $V=(d / d t)_{t=0}\left(x, w_{\ell t}\right)$ with $w_{\ell 0}=w_{\ell}$. Then we have

$$
\begin{aligned}
& \left(\pi^{*} \alpha_{S O(3)}\right)^{a \ell}(V)=\alpha_{S O(3)}\left((d / d t)_{t=0} \varphi_{a}^{-1}\left(x, \xi_{1}^{q}\left(f_{\ell}\left(w_{\ell t}\right)\right)\right)\right) \\
& \quad=\alpha_{S O(3)}\left((d / d t)_{t=0}\left[\varphi_{a}^{-1}\left(x, \xi_{1}^{q}\left(f_{\ell}\left(w_{\ell}\right)\right)\right) \cdot \xi_{1}^{q}\left(f_{\ell}\left(w_{\ell}\right)\right)^{-1} \xi_{1}^{q}\left(f_{\ell}\left(w_{\ell t}\right)\right)\right]\right) \\
& \quad=\xi_{1}^{q}\left(f_{\ell}\left(w_{\ell}\right)\right)^{-1} d\left(\xi_{1}^{q} \circ f_{\ell}\right)(V) .
\end{aligned}
$$

Observing the local trivializations of $P_{\operatorname{Spin}^{c}(n)}(Z)$ given in $\S 2$ and those of $\pi^{*} P_{\operatorname{Spin}^{q}(n)}$ given above, we know that we have a reduction embedding $P_{\operatorname{Spin}^{c}(n)}(Z) \equiv P_{\operatorname{Spin}^{q}(n)} \hookrightarrow \pi^{*} P_{\operatorname{Spin}^{q}(n)}, p_{x} \mapsto\left(\left[p_{x},[1]\right], p_{x}\right) \quad([14$, Lemma $1.3])$, which, combined with the canonical embedding $U(1)=S O(2) \hookrightarrow$ $S O(3)$, induces a reduction embedding

$$
P_{U(1)}^{\mathcal{H}}(Z)=P_{\operatorname{Spin}^{c}(n)}(Z) \times_{\xi_{1}^{c}} U(1) \hookrightarrow \pi^{*} P_{S O(3)} .
$$

The Lie algebra $\mathfrak{u}(1)=\mathfrak{s o}(2)$ has $v_{2} \wedge v_{3}$ as a basis. Hence, if we define $\mathfrak{m}$ to be the subspace of $\mathfrak{s o}(3)$ spanned by $\left\{v_{3} \wedge v_{1}, v_{1} \wedge v_{2}\right\}$, we have a decomposition $\mathfrak{s o}(3)=\mathfrak{u}(1) \oplus \mathfrak{m}$ with $\operatorname{Ad}(U(1)) \mathfrak{m} \subset \mathfrak{m}$. Now we can rigorously say that the connection $\alpha_{U(1)}^{\mathcal{H}}$ on $P_{U(1)}^{\mathcal{H}}(Z)$ is just the $\mathfrak{u}(1)$-component of 
$\pi^{*} \alpha_{S O(3)}$ restricted to it. Let us denote by $\left(\alpha_{U(1)}^{\mathcal{H}}\right)^{a \ell}$ its connection forms associated to the local trivializations $P_{U(1)}^{\mathcal{H}}(Z) \mid U_{a \ell} \cong U_{a} \times W_{\ell} \times U(1)$, $\left[p_{x}, 1\right]\left(\leftrightarrow\left[\left[p_{x},[1]\right], p_{x}\right]\right) \leftrightarrow\left(x, w_{\ell}, f_{\ell}\left(w_{\ell}\right)^{-1} \tilde{f}_{a}\left(p_{x}\right)\right)$.

LeMma 3.2. $\quad\left(\alpha_{U(1)}^{\mathcal{H}}\right)^{a \ell}=\sqrt{-1}\left[\left(\pi^{*} \alpha_{S O(3)}\right)^{a \ell}\right]_{32}$

Proof. Take the cross-section $\sigma_{a \ell}: U_{a} \times W_{\ell} \rightarrow P_{U(1)}^{\mathcal{H}}(Z) \mid U_{a \ell}, \sigma_{a \ell}\left(x, w_{\ell}\right)$ $=\left(x, w_{\ell}, 1\right) \in U_{a} \times W_{\ell} \times U(1) \cong P_{U(1)}^{\mathcal{H}}(Z) \mid U_{a \ell}$. Then we have

$$
\left(\alpha_{U(1)}^{\mathcal{H}}\right)^{a \ell}=\sigma_{a \ell}^{*} \alpha_{U(1)}^{\mathcal{H}}=\sqrt{-1}\left[\left(\pi^{*} \alpha_{S O(3)}\right)^{a \ell}\right]_{32} .
$$

Now, let $\nabla^{\mathbf{L}_{\mathcal{H}}}$ be the covariant derivative on $\mathbf{L}_{\mathcal{H}}$ associated to $\alpha_{U(1)}^{\mathcal{H}}$.

Proposition 3.3. The connection forms of $\nabla^{\mathbf{L}_{\mathcal{H}}}$ associated to the local cross-sections $U_{a} \times W_{\ell} \rightarrow \mathbf{L}_{\mathcal{H}},\left(x, w_{\ell}\right) \mapsto\left[\left(x, w_{\ell}\right), 1\right]$ (see $\left.(2.7)\right)$, are

$$
\begin{aligned}
-\frac{(-1)^{\ell} \sqrt{-1}}{1+\left|w_{\ell}\right|^{2}}\left\{\left(\left|w_{\ell}\right|^{2}-1\right) \alpha_{32}^{a}+2 \operatorname{Im} w_{\ell} \alpha_{13}^{a}+\right. & \left.(-1)^{\ell} 2 \operatorname{Re} w_{\ell} \alpha_{21}^{a}\right\} \\
& -\frac{w_{\ell} d \bar{w}_{\ell}-\bar{w}_{\ell} d w_{\ell}}{1+\left|w_{\ell}\right|^{2}} .
\end{aligned}
$$

Proof. Through the isomorphism ad $=\operatorname{Ad}_{*}: \mathfrak{s p}(1) \cong \mathfrak{s o}(3)$ we may change $\alpha_{S O(3)}, \alpha_{S O(3)}^{a}$ into $\alpha_{S p(1)}, \alpha_{S p(1)}^{a}$ which take values in $\mathfrak{s p}(1)$. We have certainly

$$
\alpha_{S p(1)}^{a}=\alpha_{32}^{a} \frac{\mathbf{i}}{2}+\alpha_{13}^{a} \frac{\mathbf{j}}{2}+\alpha_{21}^{a} \frac{\mathbf{k}}{2} .
$$

Lemma 3.2 says

$$
\left(\alpha_{U(1)}^{\mathcal{H}}\right)^{a \ell}=\sqrt{-1}\left[\left(\pi^{*} \alpha_{S p(1)}\right)^{a \ell}\right]_{\mathbf{i} / 2}
$$

and Lemma 3.1 asserts

$$
\begin{aligned}
& \left(\pi^{*} \alpha_{S p(1)}\right)^{a \ell}(X)=\rho_{\ell}\left(w_{\ell}\right)_{*}^{-1} \alpha_{S p(1)}^{a}(X), \\
& \left(\pi^{*} \alpha_{S p(1)}\right)^{a \ell}(V)=\rho_{\ell}\left(w_{\ell}\right)_{*}^{-1} d \rho_{\ell}(V) .
\end{aligned}
$$

These induce the first and second terms of (3.5) respectively. Let us show it in the case $\ell=0$. Set $w_{0}=z$. Then we have

$$
\rho_{0}\left(w_{0}\right)_{*}^{-1} \alpha_{S p(1)}^{a}=\frac{1-\mathbf{j} z}{\sqrt{1+|z|^{2}}} \alpha_{S p(1)}^{a} \frac{1+\mathbf{j} z}{\sqrt{1+|z|^{2}}}
$$




$$
\begin{aligned}
=\frac{1}{1+|z|^{2}}\left\{\frac{\alpha_{32}^{a}}{2}\left(-\left(|z|^{2}-1\right) \mathbf{i}+2 \bar{z} \mathbf{k}\right)\right. & \\
+ & \frac{\alpha_{13}^{a}}{2}\left(-(z-\bar{z})+\left(1+\bar{z}^{2}\right) \mathbf{j}\right) \\
& \left.+\frac{\alpha_{21}^{a}}{2}\left(-(z+\bar{z}) \mathbf{i}+\left(1-\bar{z}^{2}\right) \mathbf{k}\right)\right\} .
\end{aligned}
$$

Hence $\sqrt{-1}\left[\rho_{0}\left(w_{0}\right)_{*}^{-1} \alpha_{S p(1)}^{a}\right]_{\mathbf{i} / 2}$ induces the first term in (3.5) with $\ell=0$. Moreover, we have

$$
\rho_{0}\left(w_{0}\right)_{*}^{-1} d \rho_{0}=\frac{1-\mathbf{j} z}{\sqrt{1+|z|^{2}}} d\left(\frac{1+\mathbf{j} z}{\sqrt{1+|z|^{2}}}\right)=-\frac{z d \bar{z}-\bar{z} d z-2 \mathbf{j} d z}{2\left(1+|z|^{2}\right)} .
$$

Hence $\sqrt{-1}\left[\rho_{0}\left(w_{0}\right)_{*}^{-1} d \rho_{0}\right]_{\mathbf{i} / 2}$ induces the second term in $(3.5)$ with $\ell=0$. Thus we get the proposition.

\section{$\S 4$. Spinor bundles and Dirac operators}

Let $n$ be odd from now on throughout the paper. We take the complex spinor representation $\Delta: \operatorname{Spin}(n+2) \rightarrow G L_{\mathbb{C}}(S)\left(\operatorname{dim} S=2^{(n+1) / 2}\right)$ and the standard one $r_{C}: U(1) \rightarrow G L_{\mathbb{C}}(\mathbb{C}) \equiv G L_{\mathbb{C}}(C)$, which induce spinor bundles

$$
\begin{aligned}
\mathbf{S} & =P_{\operatorname{Spin}(n+2)}(Z) \times_{\Delta} S \\
\mathbf{S}^{c} & =P_{\operatorname{Spin}^{c}(n+2)}(Z) \times_{\Delta} \otimes r_{C} \\
& S \otimes C \quad\left(\cong \mathbf{S} \otimes \mathbf{U}_{Z} \otimes \mathbf{U}_{Z}^{*} \cong \mathbf{S}\right) .
\end{aligned}
$$

Here the isomorphisms in the parenthesis are due to (2.11) and (2.14). The Ehresmann connections $\alpha^{\mathbf{S}}=\xi^{*} \alpha^{Z}, \alpha^{\mathbf{S}^{c}}=\xi^{c *}\left(\alpha^{Z} \oplus\left(\alpha_{U(1)}^{\mathcal{H}} \otimes 1+1 \otimes \alpha_{U(1)}^{\mathcal{V}}\right)\right)$ induce covariant derivatives $\nabla^{\mathbf{S}}, \nabla^{\mathbf{S}^{c}}$ on (4.1), which define Dirac operators

$$
D=\sum e_{a} \circ \nabla_{e_{a}}^{\mathbf{S}}, \quad D^{c}=\sum e_{a} \circ \nabla_{e_{a}}^{\mathbf{S}^{c}},
$$

where $\left\{e_{a}\right\}$ are local orthonormal basis of $T Z$ and $e_{a} \circ$ means the Clifford action of $e_{a}$.

Let us replace $\alpha^{Z}$ by a separated connection $\alpha^{Z, \oplus}=\pi^{*} \alpha^{M} \oplus \alpha^{\mathcal{V}}$, which defines other covariant derivatives $\nabla^{\mathbf{S}, \oplus}, \nabla^{\mathbf{S}^{c}, \oplus}$ on (4.1). First we will investigate the separated covariant derivatives, which are surely simpler in construction than $\nabla^{\mathbf{S}}, \nabla^{\mathbf{S}^{c}}$ which we really want to understand. Denote by $\mathbf{S}_{\mathcal{H}}^{(c)}, \mathbf{S}_{\mathcal{V}}^{(c)}=\mathbf{S}_{\mathcal{V}}^{(c)+} \oplus \mathbf{S}_{\mathcal{V}}^{(c)-}$ the (locally defined) spinor bundles associated to $P_{\operatorname{Spin}^{(c)}(n)}(Z), P_{\operatorname{Spin}^{(c)}(2)}^{\mathcal{V}}(Z)$. Here the splitting of $\mathbf{S}_{\mathcal{V}}^{(c)}$ is given by 
the usual splitting of spinor representation $\Delta_{2}=\Delta_{2}^{+} \oplus \Delta_{2}^{-}: \operatorname{Spin}(2) \rightarrow$ $G L_{\mathbb{C}}\left(S_{2}\right)=G L_{\mathbb{C}}\left(S_{2}^{+} \oplus S_{2}^{-}\right)$. Let us attach to them the covariant derivatives $\nabla^{\mathbf{S}_{\mathcal{H}}^{(c)}}, \nabla^{\mathbf{S}_{\mathcal{V}}^{(c)}}$ associated to the Ehresmann connections $\alpha^{\mathbf{S}_{\mathcal{H}}}=\xi^{*}\left(\pi^{*} \alpha^{M}\right)$, $\alpha^{\mathbf{S}_{\mathcal{H}}^{c}}=\xi^{c *}\left(\pi^{*} \alpha^{M} \oplus \alpha_{U(1)}^{\mathcal{H}}\right), \alpha^{\mathbf{S}_{\mathcal{V}}}=\xi^{*} \alpha^{\mathcal{V}}, \alpha^{\mathbf{S}_{\mathcal{V}}^{c}}=\xi^{c *}\left(\alpha^{\mathcal{V}} \oplus \alpha_{U(1)}^{\mathcal{V}}\right)$. On the other hand, we may attach to $\mathbf{U}_{Z}$ two kinds of covariant derivatives $\nabla^{\mathbf{U}, \mathcal{V}}, \nabla^{\mathbf{U}, \mathcal{H}}$ which are associated to $\alpha_{U(1)}^{\mathcal{V}}, \alpha_{U(1)}^{\mathcal{H}}$ through the identifications $\mathbf{L}_{\mathcal{V}} \cong K_{Z}^{*} \cong \mathbf{U}_{Z}^{*} \otimes \mathbf{U}_{Z}^{*}$ and $\mathbf{L}_{\mathcal{H}} \cong K_{Z} \cong \mathbf{U}_{Z} \otimes \mathbf{U}_{Z}$ given in $\S 3$. In the case we need to specify which one is attached, we use the expressions $\mathbf{U}_{Z}\left(\nabla^{\mathbf{U}, \mathcal{V}}\right)$ etc. Now it will be obvious that (2.11) and (2.14) induce the identifications including covariant derivatives

$$
\begin{aligned}
\mathbf{S}\left(\nabla^{\mathbf{S}, \oplus}\right) & =\mathbf{S}_{\mathcal{H}} \otimes \mathbf{S}_{\mathcal{V}}=\mathbf{S}_{\mathcal{H}} \otimes\left(\mathbf{S}_{\mathcal{V}}^{c} \otimes \mathbf{U}_{Z}\left(\nabla^{\mathbf{U}, \mathcal{V}}\right)\right), \\
\mathbf{S}^{c}\left(\nabla^{\mathbf{S}^{c}, \oplus}\right) & =\mathbf{S}_{\mathcal{H}}^{c} \otimes \mathbf{S}_{\mathcal{V}}^{c}=\mathbf{S}_{\mathcal{H}} \otimes\left(\mathbf{S}_{\mathcal{V}}^{c} \otimes \mathbf{U}_{Z}\left(\nabla^{\mathbf{U}, \mathcal{H}}\right)\right) .
\end{aligned}
$$

Namely, the difference between $\nabla^{\mathbf{S}, \oplus}$ and $\nabla^{\mathbf{S}^{c}, \oplus}$ lies only in covariant derivatives on $\mathbf{S}_{\mathcal{V}}=\mathbf{S}_{\mathcal{V}}^{c} \otimes \mathbf{U}_{Z}$

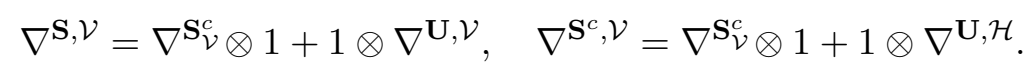

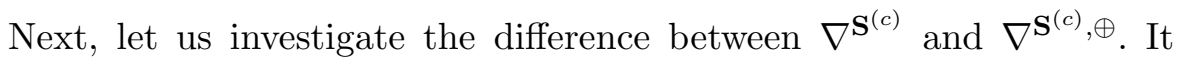
will be clear that the difference comes from the difference between the covariant derivatives $\nabla^{Z}$ and $\nabla^{Z, \oplus}=\pi^{*} \nabla^{M} \oplus \nabla^{\mathcal{V}}$ on $T Z$. Eventually, if we

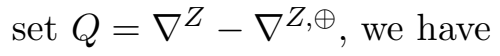

$$
\nabla_{e_{a}}^{\mathbf{S}^{(c)}}=\nabla_{e_{a}}^{\mathbf{S}^{(c)}, \oplus}+\frac{1}{4} \sum_{b, c} g^{Z}\left(Q\left(e_{a}\right) e_{b}, e_{c}\right) e_{b^{\circ} e_{c^{\circ}}}
$$

Refer to $[5,(4.24)]$. Let us denote by $T$ the torsion tensor of $\nabla^{Z, \oplus}$, which is compatible with the metric $g^{Z}$. Then [14, Lemma 2.1(3)] says that, for horizontal vectors $X, Y$ and a vertical vector $U$, we have

$$
\begin{aligned}
g^{Z}(Q(X) U, Y) & =-g^{Z}(Q(X) Y, U) \\
& =g^{Z}(Q(U) X, Y)=\frac{1}{2} g^{Z}(T(X, Y), U),
\end{aligned}
$$

and $g^{Z}(Q(\cdot) \cdot, \cdot)$ vanishes for all other combinations of horizontal and vertical vectors. We take now a local orthonormal frame $\left(e_{1}, \cdots, e_{n}\right)=\left(e_{1}^{\prime}, \cdots, e_{n}^{\prime}\right)$ of $T M$ and lift it to $Z$ dented by the same symbol, and, moreover, take such a frame $\left(e_{n+1}, e_{n+2}\right)=\left(e_{1}^{\prime \prime}, e_{2}^{\prime \prime}\right)$ of $\mathcal{V}$. Accordingly we have

$$
\sum_{a, b, c} g^{Z}\left(Q\left(e_{a}\right) e_{b}, e_{c}\right) e_{a} \circ e_{b} \circ e_{c} \circ=-\frac{1}{2} \sum_{i, j, k} g^{Z}\left(T\left(e_{i}^{\prime}, e_{j}^{\prime}\right), e_{k}^{\prime \prime}\right) e_{k}^{\prime \prime} \circ e_{i}^{\prime} \circ e_{j}^{\prime} \circ
$$




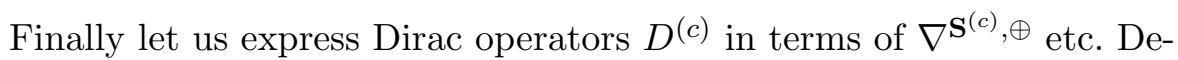
note by $\mathbf{S}_{M}$ the locally defined spinor bundle over $M$ associated to $P_{\operatorname{Spin}(n)}$. Then $\mathbf{S}_{\mathcal{H}}$ may be naturally identified with $\pi^{*} \mathbf{S}_{M}$ because of (2.10). Thus we obtain the tensor product expression

$$
\Gamma\left(\mathbf{S}^{(c)}\right)=\pi^{*} \Gamma\left(\mathbf{S}_{M}\right) \otimes \Gamma\left(\mathbf{S}_{\mathcal{V}}^{c} \otimes \mathbf{U}_{Z}\right)
$$

Consider the operators acting on the right side, $\nabla^{\mathbf{S}^{(c)}, \oplus}$ and

$$
\begin{aligned}
D^{\mathbf{S}^{(c)}, \mathcal{V}} & =1 \otimes D^{\mathbf{S}^{(c)}, \mathcal{V}}=1 \otimes \sum e_{k}^{\prime \prime} \nabla_{e_{k}^{\prime \prime}}^{\mathbf{S}^{(c)}, \mathcal{V}}, \\
c(T) & =\sum_{i \leq j} e_{i}^{\prime} \circ e_{j}^{\prime} \circ c(T)\left(e_{i}^{\prime}, e_{j}^{\prime}\right)=\sum_{i \leq j} e_{i}^{\prime} \circ e_{j}^{\prime} \circ \sum_{k} g^{Z}\left(T\left(e_{i}^{\prime}, e_{j}^{\prime}\right), e_{k}^{\prime \prime}\right) e_{k}^{\prime \prime} .
\end{aligned}
$$

LEMMA 4.1. $\quad D^{(c)}=\sum e_{i}^{\prime} \circ \nabla_{e_{i}^{\prime}}^{\mathbf{S}^{(c)}, \oplus}+D^{\mathbf{S}^{(c)}, \mathcal{V}}-\frac{1}{4} c(T)$

Proof. (4.5) and (4.7) imply

$$
D^{(c)}=\sum e_{a} \circ \nabla_{e_{a}}^{\mathbf{S}^{(c)}, \oplus}-\frac{1}{4} c(T) .
$$

Hence, using the fact $\nabla_{e_{k}^{\prime \prime}}^{\mathbf{S}^{(c)}, \oplus}=1 \otimes \nabla_{e_{k}^{\prime \prime}}^{\mathbf{S}^{(c)}, \mathcal{V}}$, we obtain the lemma.

\section{$\S 5$. Proof of Theorem 2}

First of all, let us recall the so-called $\eta$-invariant. The $\eta$-function of a Dirac operator $D$ is defined by

$$
\eta(D)(s)=\frac{1}{\Gamma((s+1) / 2)} \int_{0}^{\infty} t^{(s-1) / 2} \operatorname{Tr}\left(D e^{-t D^{2}}\right) d t, \quad \operatorname{Re} s \gg 0 .
$$

By analytic continuation to the whole complex plane we obtain a meromorphic function, which is regular at $s=0([1])$. The $\eta$-invariant of $D$ is the value at $s=0$, i.e.,

$$
\eta(D)=\eta(D)(0)=\frac{1}{\sqrt{\pi}} \int_{0}^{\infty} t^{-1 / 2} \operatorname{Tr}\left(D e^{-t D^{2}}\right) d t .
$$

Note that $\operatorname{Tr}\left(D e^{-t D^{2}}\right)=O\left(t^{1 / 2}\right)$ as $t \rightarrow 0([6,(2.13)])$ so that the above integral expression for $\eta(D)$ is well-defined.

We will investigate the limiting behavior of $\eta\left(D_{\varepsilon}\right)$ in the following. It is easy to modify it for $\eta\left(D_{\varepsilon}^{c}\right)$. 
We begin with finding out an expression for $D_{\varepsilon}$ similar to Lemma 4.1. Rigorously to say, the adiabatic version $D_{\varepsilon}$ is constructed as follows: The reduced structure bundle $P_{S O(n)}\left(M, \varepsilon^{-1} g^{M}\right)$ of $\left(M, \varepsilon^{-1} g^{M}\right)$ is canonically isomorphic to the original one $P_{S O(n)}$ (associated to $g^{M}$ ) by the identification $\left(\varepsilon^{1 / 2} e_{1}, \cdots, \varepsilon^{1 / 2} e_{n}\right) \leftrightarrow\left(e_{1}, \cdots, e_{n}\right)$, and hence has a canonical $\operatorname{Spin}^{q}(n)$-bundle $P_{\operatorname{Spin}^{q}(n)}\left(Z, g_{\varepsilon}^{Z}\right)$, which defines naturally a $\operatorname{Spin}(n+2)$ bundle $P_{\operatorname{Spin}(n+2)}\left(Z, g_{\varepsilon}^{Z}\right)$ in the similar way as in $\S 2$. Note that the metric $g^{\mathcal{V}}$ is not changed. We have then the associated spinor bundle

$$
\mathbf{S}\left(g_{\varepsilon}^{Z}\right)=P_{\operatorname{Spin}(n+2)}\left(Z, g_{\varepsilon}^{Z}\right) \times{ }_{\Delta} S .
$$

The Levi-Civita connection $\alpha_{\varepsilon}^{Z}$ associated to $g_{\varepsilon}^{Z}$ induces its covariant derivative $\nabla^{\mathbf{S}\left(g_{\varepsilon}^{Z}\right)}$, which defines the desired Dirac operator $D_{\varepsilon}$. Thus (5.3) with Clifford multiplication ${ }_{{ }_{\varepsilon}}$, on the cross-sections of which $D_{\varepsilon}$ acts, varies according to $\varepsilon$ unfortunately. This is quite troublesome. Through the following identifications, however, we may regard $D_{\varepsilon}$ as acting on $\Gamma(\mathbf{S})$ which does not vary. Let us naturally identify $P_{S O(n+2)}(Z)$ and $P_{S O(n+2)}\left(Z, g_{\varepsilon}^{Z}\right)$ $\left(\left(e_{1}, \cdots, e_{n+2}\right) \leftrightarrow\left(\varepsilon^{1 / 2} e_{1}, \cdots, \varepsilon^{1 / 2} e_{n}, e_{n+1}, e_{n+2}\right)\right)$. Accordingly the Clifford bundles $C l(T M)$ and $C l\left(T M, g_{\varepsilon}^{Z}\right)$ are identified and, finally, the spinor bundles $\mathbf{S}$ and $\mathbf{S}\left(g_{\varepsilon}^{Z}\right)$ are canonically identified. Simply to say, we have only to identify $\left(g^{Z}, e_{i}^{\prime} \circ, e_{j}^{\prime \prime} \circ\right)$ and $\left(g_{\varepsilon}^{Z}, \varepsilon^{1 / 2} e_{i}^{\prime}{ }^{\circ} \varepsilon, e_{j}^{\prime \prime}{ }^{\circ} \varepsilon\right)$. Now it will be clear that $D_{\varepsilon}$ regarded as acting on $\Gamma(\mathbf{S})$ has a following expression using operators acting on the right side of (4.8):

$$
D_{\varepsilon}=\varepsilon^{1 / 2} \sum e_{i}^{\prime} \circ \nabla_{e_{i}^{\prime}}^{\mathbf{S}, \oplus}+D^{\mathbf{S}, \mathcal{V}}-\frac{\varepsilon}{4} c(T) .
$$

Next, let us define a locally defined infinite dimensional vector bundle $H_{\infty}=H_{\infty}^{+} \oplus H_{\infty}^{-}$over $M$ by setting at $x \in M$

$$
H_{\infty,(x)}^{ \pm}=\Gamma\left(\mathbf{S}_{\mathcal{V},(x)}^{c \pm} \otimes \mathbf{U}_{Z,(x)}\right)
$$

where $\mathbf{S}_{\mathcal{V},(x)}^{c \pm}$ etc. are the restrictions of $\mathbf{S}_{\mathcal{V}}^{c \pm}$ etc. to the fibre $\pi^{-1}(x)$. We have an obvious functorial isomorphism

$$
\Gamma\left(\mathbf{S}_{\mathcal{V}}^{c} \otimes \mathbf{U}_{Z}\right) \cong \Gamma\left(H_{\infty}\right), \quad \psi \leftrightarrow \tilde{\psi}, \quad \tilde{\psi}(x)=\left(\pi^{-1}(x) \ni z \mapsto \psi(z)\right),
$$

which induces a hermitian fibre metric $\left(\tilde{\psi}_{1}, \tilde{\psi}_{2}\right)_{x}=\int_{\pi^{-1}(x)}\left(\psi_{1}(z), \psi_{2}(z)\right)$. $\operatorname{vol}_{\mathcal{V}_{x}}(z)$ on $H_{\infty}$. Here $\left(\psi_{1}(z), \psi_{2}(z)\right)$ is the pointwise hermitian inner product at $z \in \pi^{-1}(x)$ and $\operatorname{vol}_{\mathcal{V}_{x}}$ is the volume element of $\pi^{-1}(x)$. We take now 
a superconnection on $H_{\infty}$

$$
B_{t}=\tilde{\nabla}^{\mathbf{S}, \mathcal{V}}+t^{1 / 2} D^{\mathbf{S}, \mathcal{V}}-\frac{1}{4 t^{1 / 2}} \hat{c}(T), \quad t>0
$$

where we set $\tilde{\nabla}_{e_{i}^{\prime}}^{\mathbf{S}, \mathcal{V}} \tilde{\psi}=\left(\nabla_{e_{i}^{\prime}}^{\mathbf{S}, \mathcal{V}} \psi\right)^{\sim}$ and $\hat{c}(T)=\sum_{i \leq j} e_{i}^{\prime *} \wedge e_{j}^{* *} \wedge \otimes c(T)\left(e_{i}^{\prime}, e_{j}^{\prime}\right)(\mathrm{a}$ $C l(\mathcal{V})$-valued 2 -form on $M)$. Note that this is unitary with respect to the hermitian fibre metric.

Let us consider an obvious functorial isomorphism $(5.8) \pi^{*} \Gamma\left(\mathbf{S}_{M}\right) \otimes \Gamma\left(\mathbf{S}_{\mathcal{V}}^{c} \otimes \mathbf{U}_{Z}\right) \cong \Gamma\left(\mathbf{S}_{M} \otimes H_{\infty}\right), \quad \pi^{*} \phi \otimes \psi \leftrightarrow \phi \otimes \tilde{\psi}$ and let $\nabla^{\mathbf{S}_{M}}$ be the covariant derivative on $\mathbf{S}_{M}$ associated to $\xi^{*} \alpha^{M}$. We may take then a superconnection

$$
\mathbf{B}_{t}=\nabla^{\mathbf{S}_{M}} \otimes 1+1 \otimes B_{t}
$$

on $\mathbf{S}_{M} \otimes H_{\infty}$. It is easily shown that the "Dirac operator" (for $\mathbf{S}_{M} \otimes H_{\infty}$ ) associated to $\varepsilon^{1 / 2} \mathbf{B}_{1 / \varepsilon}$, i.e., its quantization (replacing $e_{i}^{\prime *} \wedge, e_{i}^{* *} \wedge e_{j}^{* *}$ by $e_{i}^{\prime}$, $\left.e_{i}^{\prime} \circ e_{j}^{\prime} \circ\right)$, may be identified with our Dirac operator $D_{\varepsilon}$ through (4.8) and (5.8). The superconnection (5.7) was taken so that it fits such a framework $([5,6])$.

$H_{\infty}$ is obtained by "pasting" $\left\{U_{a} \times \Gamma\left(\mathbf{S}_{\mathbb{C} P^{1}}^{c} \otimes \mathbf{U}_{\mathbb{C} P^{1}}\right)\right\}_{a}$ together using the pseudo-transition functions $\left\{\tilde{f}_{0 b a}\right\}$, where $\mathbf{S}_{\mathbb{C} P^{1}}^{c}$ is a spinor bundle associated to the canonical Spin ${ }^{c}$ structure of $\mathbb{C} P^{1}$ and $\mathbf{U}_{\mathbb{C} P^{1}}$ is the universal line bundle over $\mathbb{C} P^{1}$, so that it has ambiguity in the sense that the "pasting" using $\tilde{f}_{0 b a}$ may differ in sign from the roundabout "pasting" using $\tilde{f}_{0 b c} \tilde{f}_{0 c a}$. But such ambiguity does not appear in the "connection forms" $\left(\in \Gamma\left(\operatorname{End}\left(\mathbf{S}_{\mathbb{C} P^{1}}^{c} \otimes \mathbf{U}_{\mathbb{C} P^{1}}\right) \otimes \wedge^{1} T^{*} U_{a}\right)\right)$ of the locally defined $\tilde{\nabla} \mathbf{S}, \mathcal{V}$. In the sense, we may think of $\tilde{\nabla}^{\mathbf{S}}, \mathcal{V}$ as being globally defined. As for the remained terms $B_{[0]}=D^{\mathbf{S}, \mathcal{V}}$ and $B_{[2]}=-4^{-1} \hat{c}(T)$ in (5.7), certainly they exist globally. Namely, the bundle End $\left(H_{\infty}\right)$ exists globally so that $B_{[i]}$ is a global cross-section of the globally defined vector bundle $\wedge^{i} T^{*} M \otimes$ End $\left(H_{\infty}\right)([3$, Proposition 1.39]),

$$
B_{[i]} \in \Gamma\left(M, \wedge^{i} T^{*} M \otimes \operatorname{End}\left(H_{\infty}\right)\right) .
$$

Further, we may think of the curvature $B_{t}^{2}$ as being (the operator given by) an element of $\Gamma\left(M, \wedge T^{*} M \otimes\right.$ End $\left.\left(H_{\infty}\right)\right)$ so that the heat operator $e^{-B_{t}^{2}}$ can be regarded also as (the operator given by) an element of it ([3, Proposition $1.38])$,

$$
e^{-B_{t}^{2}} \in \Gamma\left(M, \wedge T^{*} M \otimes \operatorname{End}\left(H_{\infty}\right)\right)
$$


LEMma 5.1. The fibrewise supertrace $\operatorname{str}\left(e^{-B_{t}^{2}}\right)=\operatorname{tr}\left(e^{-B_{t}^{2}} \mid H_{\infty}^{+}\right)-$ $\operatorname{tr}\left(e^{-B_{t}^{2} \mid H_{\infty}^{-}}\right)$, called the (renormalized) Chern character form of $B_{t}$ in [3], is a globally defined even degree form on $M$. Further we have

$$
\begin{aligned}
& \lim _{t \rightarrow \infty} \operatorname{str}\left(e^{-B_{t}^{2}}\right)=0 \\
& \lim _{t \rightarrow 0} \operatorname{str}\left(e^{-B_{t}^{2}}\right)=(2 \pi \sqrt{-1})^{-1} \int_{Z / M} \hat{\mathbb{A}}\left(2 \pi \sqrt{-1} \Omega^{\mathcal{V}}\right) .
\end{aligned}
$$

Proof. We set $\operatorname{End}^{j}\left(H_{\infty}\right)=\operatorname{Hom}\left(H_{\infty}^{ \pm}, H_{\infty}^{ \pm}\right)($if $j=0), \operatorname{Hom}\left(H_{\infty}^{ \pm}, H_{\infty}^{\mp}\right)$ (if $j=1$ ), and say that the elements of $\Gamma\left(M, \wedge^{i} T^{*} M \otimes \operatorname{End}^{j}\left(H_{\infty}\right)\right)$ are of total degree $i+j$. Then $B_{t}^{2}$ is of even total degree and so is the heat operator $e^{-B_{t}^{2}}$. Hence $\operatorname{str}\left(e^{-B_{t}^{2}}\right)$ is an even degree form. In order to prove (5.12), let us regard $D^{\mathbf{S}, \mathcal{V}}$ as a family of Dirac operators along the fibres

$$
\begin{aligned}
& D^{\mathbf{S}, \mathcal{V}}=\left(D_{(x)}^{\mathbf{S}, \mathcal{V}} \mid x \in M\right), \\
& D_{(x)}^{\mathbf{S}, \mathcal{V}}=\sum e_{k}^{\prime \prime} \circ \nabla_{e_{k}^{\prime \prime}}^{\mathbf{S}, \mathcal{V}}: \Gamma\left(H_{\infty,(x)}^{( \pm)}\right) \rightarrow \Gamma\left(H_{\infty,(x)}^{(\mp)}\right) .
\end{aligned}
$$

We want to show that the index bundle Ind $D^{\mathbf{S}, \mathcal{V}}=\coprod_{x \in M} \operatorname{Ker} D_{(x)}^{\mathbf{S}, \mathcal{V}}$, which is naturally $\mathbb{Z}_{2}$-graded, is just a 0 -bundle

$$
\text { Ind } D^{\mathbf{S}, \mathcal{V}}=0
$$

It is well-known that we can identify:

$$
\begin{aligned}
\mathbf{S}_{\mathcal{V},(x)}^{c} \otimes \mathbf{U}_{Z,(x)} & =\left(\mathbf{S}_{\mathcal{V},(x)}^{c+} \otimes \mathbf{U}_{Z,(x)}\right) \oplus\left(\mathbf{S}_{\mathcal{V},(x)}^{c-} \otimes \mathbf{U}_{Z,(x)}\right) \\
& =\wedge^{0, *}\left(T_{\mathbb{C}}^{*} \mathbb{C} P^{1}\right) \otimes \mathbf{U}_{\mathbb{C} P^{1}} \\
& =\left(\wedge^{0,0}\left(T_{\mathbb{C}}^{*} \mathbb{C} P^{1}\right) \otimes \mathbf{U}_{\mathbb{C} P^{1}}\right) \oplus\left(\wedge^{0,1}\left(T_{\mathbb{C}}^{*} \mathbb{C} P^{1}\right) \otimes \mathbf{U}_{\mathbb{C} P^{1}}\right) \\
D^{\mathbf{S}, \mathcal{V}} & =\left(\begin{array}{cc}
0 & D^{\mathbf{S}, \mathcal{V},-} \\
D^{\mathbf{S}, \mathcal{V},+} & 0
\end{array}\right)=2\left(\bar{\partial}+\bar{\partial}^{*}\right)=\left(\begin{array}{cc}
0 & 2 \bar{\partial}^{*} \\
2 \bar{\partial} & 0
\end{array}\right)
\end{aligned}
$$

Here we attach to $\mathbf{S}_{\mathcal{V},(x)}^{c} \otimes \mathbf{U}_{Z,(x)}$ the covariant derivative $\nabla^{\mathbf{S}, \mathcal{V}} \mid \pi^{-1}(x)$ and attach also to $\wedge^{0, *}\left(T_{\mathbb{C}}^{*} \mathbb{C} P^{1}\right) \otimes \mathbf{U}_{\mathbb{C} P^{1}}$ the usual hermitian covariant derivative associated to $d s^{2}$. It is important that $\nabla^{\mathbf{U}, \mathcal{V}}$ restricted to the fibre $\pi^{-1}(x)$ coincides with the covariant derivative (associated to $d s^{2}$ ) of $\mathbf{U}_{\mathbb{C} P^{1}}$ so that we may think of (5.16) as an identification including such covariant derivatives (and metrics). Further $\bar{\partial}$ is the $\bar{\partial}$-operator acting on $(0, *)$-forms 
with coefficients in the holomorphic bundle $\mathbf{U}_{\mathbb{C} P^{1}}$ and $\bar{\partial}^{*}$ is its dual. On $\Gamma\left(\wedge^{0, q}\left(T_{\mathbb{C}}^{*} \mathbb{C} P^{1}\right) \otimes \mathbf{U}_{\mathbb{C} P^{1}}\right)$, we have $\operatorname{Ker} \bar{\partial}^{(*)}=\operatorname{Ker} \bar{\partial} \cap \operatorname{Ker} \bar{\partial}^{*}$, which is isomorphic further to the $q$-th Cech cohomology group $H^{q}\left(\mathbb{C} P^{1}, \mathcal{O}\left(\mathbf{U}_{\left.\mathbb{C} P^{1}\right)}\right)\right)$ with coefficients in the sheaf of holomorphic cross-sections of $\mathbf{U}_{\mathbb{C} P^{1}}$. Thus we have

$$
\operatorname{Ker} D_{(x)}^{\mathbf{S}, \mathcal{V}} \cong H^{*}\left(\mathbb{C} P^{1}, \mathcal{O}\left(\mathbf{U}_{\mathbb{C} P^{1}}\right)\right) .
$$

As is well-known, the Kodaira vanishing and the Serrè duality theorems imply that this equals $\{0\}$. Thus we obtain (5.15). Now (5.15) and [3, Corollary 9.22] imply (5.12). Next, since we can identify

$$
\mathbf{S}_{\mathcal{V}}^{c} \otimes \mathbf{U}_{Z}\left(\nabla^{\mathbf{U}, \mathcal{V}}\right) \cong \mathbf{S}_{\mathcal{V}} \otimes \mathbf{U}_{Z}^{*}\left(\nabla^{\mathbf{U}, \mathcal{V}}\right) \otimes \mathbf{U}_{Z}\left(\nabla^{\mathbf{U}, \mathcal{V}}\right) \cong \mathbf{S}_{\mathcal{V}}
$$

including covariant derivatives (see (4.3)), [3, Theorem 10.23] implies (5.13).

Making some more preparations we may prove Theorem 2. Set

$$
\begin{aligned}
& \hat{\eta}(t)=\operatorname{str}\left[\left(D^{\mathbf{S}, \mathcal{V}}+\frac{\hat{c}(T)}{4 t}\right) e^{-B_{t}^{2}}\right]=\sum[\hat{\eta}(t)]_{2 j-1}, \\
& \tilde{\eta}(t)=\sum \frac{1}{(2 \pi \sqrt{-1})^{j}}[\hat{\eta}(t)]_{2 j-1},
\end{aligned}
$$

where $[\hat{\eta}(t)]_{2 j-1}$ is the $\hat{\eta}(t)$ 's homogeneous component of degree $2 j-1$. Notice that $\left(D^{\mathbf{S}, \mathcal{V}}+\hat{c}(T) /(4 t)\right) \exp \left(-B_{t}^{2}\right)$ is of odd total degree so that $\hat{\eta}(t)$ is an odd degree form. It follows from [3, Theorems 9.23 and 10.32(1)] that we have uniform convergence

$$
\hat{\eta}(t)= \begin{cases}O\left(t^{-1}\right), & t \rightarrow \infty \\ O(1), & t \rightarrow 0\end{cases}
$$

Moreover, in the same way as the proof of $[5,(4.40)]$ it is easily proved that we have uniform convergence as $\varepsilon \rightarrow 0$

$$
\operatorname{Tr}\left(D_{\varepsilon} e^{-t D_{\varepsilon}^{2}}\right)=\sqrt{\pi} \int_{M} \hat{\mathbb{A}}\left(\Omega^{M}\right) \wedge \tilde{\eta}(t)+O\left(\varepsilon^{1 / 2}\left(1+t^{N}\right)\right)
$$

for some $N>0$.

Now let us prove Theorem 2 . 
Proof of Theorem 2 for $D_{\varepsilon}$. First, (5.15) and [5, Proposition 4.41] imply that the spectrum of $D_{\varepsilon}^{2}$ is bounded from below by some constant $\lambda_{0}>0$ for all sufficiently small $\varepsilon>0$. Hence $\lim _{\varepsilon \rightarrow 0} \eta\left(D_{\varepsilon}\right)$ exists. Moreover, (5.22) implies (0.11). Namely, the following formal computation (see (5.2)) is certified correct:

$$
\begin{aligned}
\lim _{\varepsilon \rightarrow 0} \eta\left(D_{\varepsilon}\right) & =\frac{1}{\sqrt{\pi}} \int_{0}^{\infty} t^{-1 / 2} \lim _{\varepsilon \rightarrow 0} \operatorname{Tr}\left(D_{\varepsilon} e^{-t D_{\varepsilon}^{2}}\right) d t \\
& =2 \int_{M} \hat{\mathbb{A}}\left(\Omega^{M}\right) \wedge \int_{0}^{\infty} \tilde{\eta}(t) \frac{d t}{2 t^{1 / 2}} .
\end{aligned}
$$

Here

$$
\tilde{\eta}=\int_{0}^{\infty} \tilde{\eta}(t) \frac{d t}{2 t^{1 / 2}}
$$

is convergent because of (5.21). Next, the transgression formula ([3]) for $B_{t}$ says

$$
\frac{d}{d t} \operatorname{str}\left(e^{-B_{t}^{2}}\right)=-d \frac{\hat{\eta}(t)}{2 t^{1 / 2}} .
$$

Hence, if we set $\hat{\eta}=\int_{0}^{\infty} \hat{\eta}(t) /\left(2 t^{1 / 2}\right) d t$, then (5.25) and Lemma 5.1 imply

$$
\begin{aligned}
d \hat{\eta} & =d \int_{0}^{\infty} \hat{\eta}(t) \frac{d t}{2 t^{1 / 2}}=\lim _{t \rightarrow 0} \operatorname{str}\left(e^{-B_{t}^{2}}\right)-\lim _{t \rightarrow \infty} \operatorname{str}\left(e^{-B_{t}^{2}}\right) \\
& =(2 \pi \sqrt{-1})^{-1} \int_{Z / M} \hat{\mathbb{A}}\left(2 \pi \sqrt{-1} \Omega^{\mathcal{V}}\right) .
\end{aligned}
$$

We get thus (0.12).

Proof of Theorem 2 for $D_{\varepsilon}^{c}$. Since the proof is quite similar to that of $D_{\varepsilon}$, it will suffice to explain the outline with emphasizing some points of difference. (5.4) is changed into

$$
D_{\varepsilon}^{c}=\varepsilon^{1 / 2} \sum e_{i}^{\prime} \nabla_{e_{i}^{\prime}}^{\mathbf{S}^{c}, \oplus}+D^{\mathbf{S}^{c}, \mathcal{V}}-\frac{\varepsilon}{4} c(T) .
$$

The operators on the right side act on the right side of (4.8). It is important that here the covariant derivative $\nabla^{\mathbf{U}, \mathcal{H}}$ is attached to $\mathbf{U}_{Z}$ in (4.8): see (4.3). We take a superconnection

$$
B_{t}^{c}=\tilde{\nabla}^{\mathbf{S}^{c}, \mathcal{V}}+t^{1 / 2} D^{\mathbf{S}^{c}, \mathcal{V}}-\frac{1}{4 t^{1 / 2}} \hat{c}(T), \quad t>0
$$


on $H_{\infty}^{c}=H_{\infty}$. Then we have

(5.29) $\lim _{t \rightarrow \infty} \operatorname{str}\left(e^{-\left(B_{t}^{c}\right)^{2}}\right)=0$,

(5.30) $\lim _{t \rightarrow 0} \operatorname{str}\left(e^{-\left(B_{t}^{c}\right)^{2}}\right)=(2 \pi \sqrt{-1})^{-1} \int_{Z / M} \hat{\mathbb{A}}\left(2 \pi \sqrt{-1} \Omega^{\mathcal{V}}\right)$

$$
\times \exp \left(\frac{1}{2} c_{1}\left(2 \pi \sqrt{-1}\left(\Omega^{\mathcal{V}}+\Omega^{\mathcal{H}}\right)\right)\right) .
$$

As for (5.29): Notice that the covariant derivative $\nabla^{\mathbf{S}^{c}, \mathcal{V}} \mid \pi^{-1}(x)=\left(\nabla^{\mathbf{S}_{\mathcal{V}}} \otimes\right.$ $\left.1+1 \otimes \nabla^{\mathbf{U}, \mathcal{H}}\right) \mid \pi^{-1}(x)$ is attached to $\mathbf{S}_{\mathcal{V},(x)}^{c} \otimes \mathbf{U}_{Z,(x)}$. The two covariant derivatives $\nabla^{\mathbf{U}, \mathcal{H}}$ and $\nabla^{\mathbf{U}, \mathcal{V}}$ on $\mathbf{U}_{Z}$ do not coincide with each other certainly, but these restricted to the fibres fortunately coincide because of Proposition 3.3 and Lemma 2.1, i.e.,

$$
\nabla^{\mathbf{U}, \mathcal{H}}\left|\pi^{-1}(x)=\nabla^{\mathbf{U}, \mathcal{V}}\right| \pi^{-1}(x)
$$

so that the proof of (5.12) can be seen as a proof of (5.29) with no change. As for (5.31): We have

$$
\mathbf{S}_{\mathcal{V}}^{c} \otimes \mathbf{U}_{Z}\left(\nabla^{\mathbf{U}, \mathcal{H}}\right) \cong \mathbf{S}_{\mathcal{V}} \otimes \mathbf{U}_{Z}^{*}\left(\nabla^{\mathbf{U}, \mathcal{V}}\right) \otimes \mathbf{U}_{Z}\left(\nabla^{\mathbf{U}, \mathcal{H}}\right)
$$

which corresponds to (5.19). Hence again [3, Theorem 10.23] implies (5.31). Notice that the curvature of the induced covariant derivative on $\mathbf{U}_{Z}^{*}$ differs in sign from that of $\nabla^{\mathbf{U}, \mathcal{H}}$ on $\mathbf{U}_{Z}$. Now (5.29) guarantees that the limit $\lim _{\varepsilon \rightarrow 0} \eta\left(D_{\varepsilon}^{c}\right)$ exists. Define $\tilde{\eta}^{c}(t)$ in the same way as (5.20) and put

$$
\tilde{\eta}^{c}=\int_{0}^{\infty} \tilde{\eta}^{c}(t) \frac{d t}{2 t^{1 / 2}} .
$$

Then we get (0.11) in the same way as in (5.23). Finally, the transgression formula for $B_{t}^{c},(5.29)$ and (5.31) imply (0.13).

\section{REFERENCES}

[1] M. F. Atiyah, V. K. Patodi and I. M. Singer, Spectral asymmetry and Riemannian geometry I, Math. Proc. Cambridge Philos. Soc., 77 (1975), 43-69.

[2] C. Bär, Elliptische Operatoren und Darstellungstheorie kompakter Gruppen, Bonner Mathematische Schriften, 248 (1993).

[3] N. Berline, E. Getzler and M. Vergne, Heat kernels and Dirac operators, Springer-Verlag, Berlin Heidelberg, 1992. 
[4] A. L. Besse, Einstein manifolds, Springer-Verlag, Berlin Heidelberg, 1987.

[5] J.-M. Bismut and J. Cheeger, $\eta$-invariants and their adiabatic limits, J. Amer. Math. Soc., 2 (1989), 33-70.

[6] J.-M. Bismut and D. S. Freed, The analysis of elliptic families II, Dirac operators, eta invariants and the holonomy theorem, Comm. Math. Phys., 107 (1986), 103-163.

[7] J. Cheeger, On the formulas of Atiyah-Patodi-Singer and Witten, Proc. of ICM, Berkeley (1986), 515-521.

[8] J. Cheeger, $\eta$-invariants, the adiabatic approximation and conical singularities, J. Diff. Geometry, 26 (1987), 175-221.

[9] X. Dai, Adiabatic limits, nonmultiplicativity of signature, and Leray spectral sequence, J. Amer. Math. Soc., 4 (1991), 265-321.

[10] P. Griffiths and J. Harris, Principles of algebraic geometry, John Wiley \& Sons, 1978.

[11] H. B. Lawson and M. Michelsohn, Spin geometry, Princeton Univ. Press, 1989.

[12] M. Nagase, Spin ${ }^{q}$ structures, J. Math. Soc. Japan, 47 (1995), 93-119.

[13] M. Nagase, Quaternionic symplectic manifolds and canonical quantum bundles, J. Math. Sci. Univ. Tokyo, 2 (1995), 347-374.

[14] M. Nagase, Spin ${ }^{q}$, twistor and Spin ${ }^{c}$, Comm. Math. Phys., 189 (1997), 107-126.

[15] N. R. O'Brian and J. H. Rawnsley, Twistor spaces,, Ann. Global Anal. Geom., 3 (1985), 29-58.

[16] S. M. Salamon, Quaternionic Kähler manifolds, Invent. Math., 67 (1982), 143-171.

[17] S. M. Salamon, Riemannian geometry and holonomy groups,, Longman Scientific \& Technical, 1989.

[18] E. Witten, Global gravitational anomalies, Comm. Math. Phys., 100 (1985), 197-229.

Department of Mathematics

Faculty of Science

Saitama University

Saitama, Saitama 338-8570

Japan

mnagase@rimath.saitama-u.ac.jp 